\title{
A SURVEY ON DATA ANALYSIS APPROACH FOR EFFECTIVE CONTAINMENT TOWARDS PANDEMIC INFECTIONS USING NEURAL NETWORKS
}

\author{
Anix Mary Javitha. $\mathbf{A}^{1}, \mathrm{Dr}$, Mary Livinsa. $\mathrm{Z}^{\mathbf{2}}$ \\ ${ }^{I}$ Research Scholar, Sathyabama Institute of Science and Technology, Chennai, \\ ${ }^{2}$ Associate Prof-ETCE, Sathyabama Institute of Science and Technology, Chennai
}

Article DOI: https://doi.org/10.36713/epra7553

DOI No: 10.36713/epra7553

\begin{abstract}
This Covid-19 is a new pandemic infectious era for our human life, in order to deal with it in proper manner, the necessity and importance of data analysis research reports, models, approaches are of the basic requirements for our sustainable, patience and stress less future. Neural network model organization and development with proper incorporation of pandemic condition parameters with maximum level of accuracy and efficiency are the main issues related with data analysis in pandemic infection containment. The implementation of neural networks techniques associated with data analysis approaches will be the best combination in this research area in near future.
\end{abstract}

KEYWORDS - Neural networks, Covid-19, Pandemic ,Data analysis, Containment.

\section{INTRODUCTION}

Covid-19 -The new corona virus is a respiratory virus identified in 2019 which spreads primarily through droplets generated when an infected person coughs or sneezes, or through droplets of saliva or discharge from the nose.

Prediction-A prediction is what someone thinks will happen. A prediction is a forecast, but not only about the weather. Pre means "before" and diction has to do with talking. So a prediction is a statement about the future, based on facts or evidence.

Data Analytics-Data analysis is a process of inspecting, cleansing, transforming and modeling data with the goal of discovering useful information, informing conclusions and supporting decision-making.

Neural Networks-A neural network is a series of algorithms that endeavors to recognize underlying relationships in a set of data through a process that mimics the way the human brain operates. In this sense, neural networks refer to systems of neurons, either organic or artificial in nature.
Supervised learning-It is the machine learning task of learning a function that maps an input to an output based on example input-output pairs. It infers a function from labeled training data consisting of a set of training examples[9].

Data Model-A data model is an abstract model that organizes elements of data and standardizes how they relate to one another and to the properties of real-world entities.

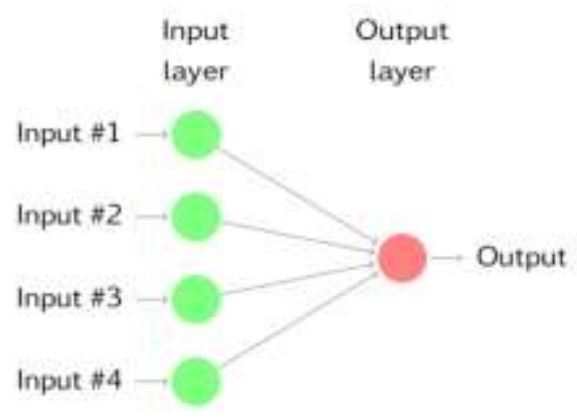

Fig-1:Neural Network Model 


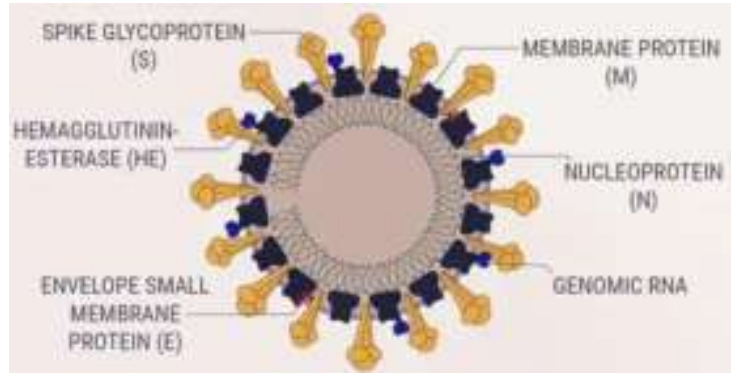

Fig-2:Covid-19 virus structure

\section{DATA ANALYSIS APPROACHES}

Data Analysis is an analytic process designed to explore data (usually large amounts of data typically business or market related) in search of consistent patterns and/or systematic relationships between variables, and then to validate the findings by applying the detected patterns to new subsets of data. The ultimate goal of data analysis is prediction - and predictive data analysis is the most common type of data exploration and one that has the most direct business applications. The process of data analysis consists of three stages: (1) the initial exploration, (2) model building or pattern identification with validation/verification, and (3) deployment (i.e., the application of the model to new data in order to generate predictions) [8].

1. Data Mining

2. Crucial Concepts in Data Mining

3. Data Warehousing

4. On-Line Analytic Processing (OLAP)

5. Exploratory Data Analysis (EDA) and Data Mining Techniques

a. EDA vs. Hypothesis Testing

b. Computational EDA Techniques

c. Graphical (data visualization)

EDA techniques

d. Verification of results of EDA

6. Neural networks

\section{APPROACHES OF NEURAL NETWORKS}

Neural networks, with their remarkable ability to derive meaning from complicated or imprecise data, can be used to extract patterns and detect trends that are too complex to be noticed by either humans or other computer techniques. A trained neural network can be thought of as an "expert" in the category of information it has been given to analyze. This expert can then be used to provide projections given new situations of interest and answer "what if" questions. Other advantages include:

1. Adaptive learning: An ability to learn how to do tasks based on the data given for training or initial experience.
2. Self-Organization: An NEURAL NETWORK can create its own organization or representation of the information it receives during learning time.

3. Real Time Operation: NEURAL NETWORK computations may be carried out in parallel, and special hardware devices are being designed and manufactured which take advantage of this capability.

4. Fault Tolerance via Redundant Information Coding: Partial destruction of a network leads to the corresponding degradation of performance. However, some network capabilities may be retained even with major network damage.

\section{COVID-19 PANDEMIC SCENARIO}

Corona virus disease (COVID-19) is an infectious disease caused by a newly discovered corona virus.

Most people infected with the COVID-19 virus will experience mild to moderate respiratory illness and recover without requiring special treatment. Older people and those with underlying medical problems like cardiovascular disease, diabetes, chronic respiratory disease, and cancer are more likely to develop serious illness [10].

The best way to prevent and slow down transmission is to be well informed about the COVID-19 virus, the disease it causes and how it spreads. Protect yourself and others from infection by washing your hands or using an alcohol based rub frequently and not touching your face.

The COVID-19 virus spreads primarily through droplets of saliva or discharge from the nose when an infected person coughs or sneezes, so it's important that you also practice respiratory etiquette (for example, by coughing into a flexed elbow).

\section{LITERATURE SURVEY ON NEURAL NETWORK ON COVID-19}

$>$ Huang $\mathrm{C}$, Wang Y, Li X, et al. "Clinical features of patients infected with 2019 novel coronavirus in Wuhan, China". Lancet 2020 describes Coronaviruses $(\mathrm{CoV})$ belong to the genus Coronavirus in the Coronaviridae. All CoVs are pleomorphic RNA viruses characteristically containing crown-shape peplomers with 80-160 nM in size and 27$32 \mathrm{~kb}$ positive polarity. With its high mutation rate, Coronaviruses are zoonotic pathogens that are present in humans and various animals with a wide range of clinical features from asymptomatic course to requirement of hospitalization in the 
intensive care unit; causing infections in respiratory, gastrointestinal, hepatic and neurologic systems.

$>$ Nada Elgendy et al "Data Analytics" describes in the information era, enormous amounts of data have become available on hand to decision makers. Big data refers to datasets that are not only big, but also high in variety and velocity, which makes them difficult to handle using traditional tools and techniques. Due to the rapid growth of such data, solutions need to be studied and provided in order to handle and extract value and knowledge from these datasets. Furthermore, decision makers need to be able to gain valuable insights from such varied and rapidly changing data, ranging from daily transactions to customer interactions and social network data.

\section{Gail Brion et al "Neural Network} Prediction of Viruses in Shellfish" describes A database was probed with neural network and multivariate logistic regression (MLR) models to investigate the efficacy of predicting PCR-identified human adenovirus (ADV), Norwalk-like virus $(\mathrm{NLV})$, and enter virus (EV) presence or absence in shellfish harvested from diverse countries in Europe (Spain, Sweden, Greece, and the United Kingdom). The relative importance of numerical and heuristic input variables to the ANN model for each country and for the combined data was analyzed with a newly defined relative strength effect, which illuminated the importance of bacteriophages as potential viral indicators.

$>$ The huge impact of Covid-19 pandemic virus spread is collected from https://www.worldometers.info/coronavirus/ \#countries.

\section{CHALLENGES IN COVID-19 DATA ANALYTICS}

Searching for relevant information sources

We are witnessing tremendous growth of articles published on this topic, already counting in thousands. For methodologists and researchers in the field of evidence synthesis, the challenge will be searching for the relevant information sources. Creating specialized, publicly accessible collection of studies with original studies about COVID-19 can surely help in this. For example, WHO has set up a collection of articles about COVID-19, compiled in a publicly available database? On March 30, 2020 this database had already included 3294 articles.

Synthesizing evidence rapidly

In a world where each day brings hundreds of new articles on a hot topic, conducting evidence synthesis will be particularly challenging. Systematic reviews are considered by many as the highest-level of evidence in the hierarchy of evidence in medicine, but their production often takes years [7]. However, multiple systematic reviews about COVID-19 have already been published. It remains to be seen what the quality of those rapidly produced systematic reviews is.

Data sharing

To enable analysis of data gathered during COVID-19 pandemic, principles of open science and raw data sharing will be of utmost importance. Global norms have been proposed [3] for data sharing during global health emergencies, and it remains to be seen whether researchers will be more likely to share their raw data publicly in articles covering COVID-19.

In conclusion, there are many methodological challenges related to producing, gathering, analyzing, reporting and publishing data in condensed timelines required during a pandemic.

\section{NEURAL NETWORK DATA IMPLEMENTATION}

Neural networks and conventional algorithmic computers are not in competition but complement each other. There are tasks are more suited to an algorithmic approach like arithmetic operations and tasks that are more suited to neural networks. Even more, a large number of tasks, require systems that use a combination of the two approaches (normally a conventional computer is used to supervise the Neural network) in order to perform at maximum efficiency.

Much is still unknown about how the brain trains itself to process information, so theories abound. In the human brain, a typical neuron collects signals from others through a host of fine structures called dendrites. The neuron sends out spikes of electrical activity through a long, thin stand known as an axon, which splits into thousands of branches. At the end of each branch, a structure called a synapse converts the activity from the axon into electrical effects that inhibit or excite activity from the axon into electrical effects that inhibit or excite activity in the connected neurons. When a neuron receives excitatory input that is sufficiently large compared with its 
inhibitory input, it sends a spike of electrical activity down its axon. Learning occurs by changing the effectiveness of the synapses so that the influence of one neuron on another changes.
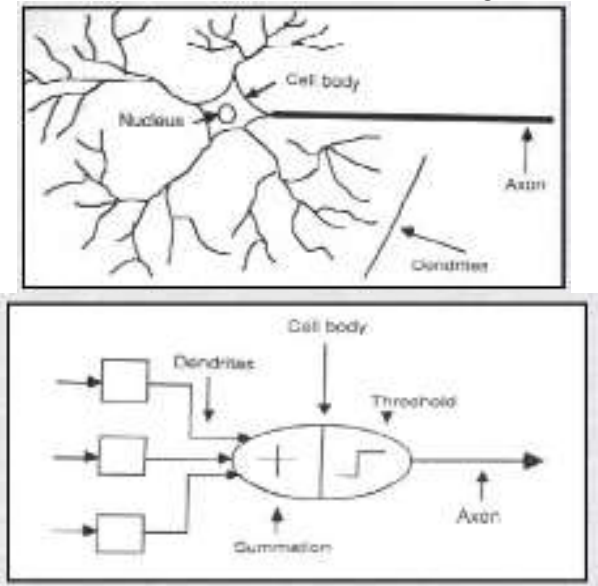

Fig-3: Artificial Neurons

An artificial neuron is a device with many inputs and one output. The neuron has two modes of operation; the training mode and the using mode. In the training mode, the neuron can be trained to fire (or not), for particular input patterns. In the using mode, when a taught input pattern is detected at the input, its associated output becomes the current output. If the input pattern does not belong in the taught list of input patterns, the firing rule is used to determine whether to fire or not [1].

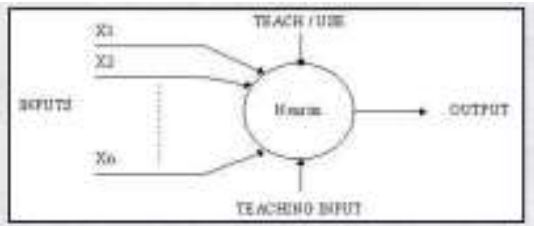

Fig-4: Training Node concept

The firing rule is an important concept in neural networks and accounts for their high flexibility. A firing rule determines how one calculates whether a neuron should fire for any input pattern. It relates to all the input patterns, not only the ones on which the node was trained. A simple firing rule can be implemented by using Hamming distance technique.

\section{NEURAL NETWORKS TOOLS PERFORMANCE}

The following table-1 illustrates the neural network tools with its implementation scope for covid-19 data analytics.

Table-1: Neural $\mathbf{n} / \mathbf{w}$ Tools for data analysis

\begin{tabular}{|l|l|}
\hline Sl.No & Software Tool name \\
\hline 1 & Neural Designer \\
\hline 2 & Neuroph,Darknet \\
\hline 3 & Keras \\
\hline 4 & NeuroSolutions \\
\hline
\end{tabular}

\begin{tabular}{|l|l|}
\hline 5 & Tflearn \\
\hline 6 & ConvNetJS \\
\hline 7 & Torch \\
\hline 8 & NVIDIA DIGITS \\
\hline 9 & Stuttgart NN Simulator \\
\hline 10 & DeepPy \\
\hline 11 & MLPNeuralNet \\
\hline 12 & DNNGraph \\
\hline 13 & AForge.Neuro \\
\hline 14 & NeuralN \\
\hline 15 & NeuralTalk2 \\
\hline 16 & Knet \\
\hline 17 & cuda-convnet2 \\
\hline 18 & DN2A \\
\hline 19 & Mocha \\
\hline 20 & HNN Lasagne neon \\
\hline 21 & LambdaNet \\
\hline 22 & gobrain \\
\hline 23 & RustNN \\
\hline 24 & deeplearn-rs \\
\hline
\end{tabular}

\section{NEURAL NETWORKS IN MEDICINE}

Neural networks are currently a 'hot' research area in medicine and it is believed that they will receive extensive application to biomedical systems in the next few years including Covid-19 with its mutations. At the moment, the research is mostly on modeling parts of the human body and recognizing diseases from various scans (e.g. cardiograms, CAT scans, ultrasonic scans, etc.) [4].

Neural networks are ideal in recognizing diseases using scans since there is no need to provide a specific algorithm on how to identify the disease. Neural networks learn by example so the details of how to recognize the disease are not needed. What is needed is a set of examples that are representative of all the variations of the disease. The quantity of examples is not as important as the 'quantity'. The examples need to be selected very carefully if the system is to perform reliably and efficiently [5].

\section{APPLICATIONS OF NEURAL NETWORKS}

1. Neural networks are best at identifying patterns or trends in data, they are well suited for prediction or forecasting needs including [6]:

$>$ Sales forecasting

$>$ Industrial process control

$>$ Customer research

$>$ Data validation

$>$ Risk management

$>$ Target marketing

$>$ Pandemic data analysis and prediction 
Neural Networks is also used in the following specific paradigms: recognition of speakers in communications; diagnosis of hepatitis; recovery of telecommunications from faulty software; interpretation of multi meaning Chinese words; undersea mine detection; texture analysis; threedimensional object recognition; hand-written word recognition; and facial recognition and pandemic disease data analysis and prediction.

\section{CONCLUSION}

Neural network in information technology enhances the information provided on utility sites to be structured. This allows for a pandemic infection data analysis of the covid-19 Web data domains to access specific information without having to search the entire site. With the use of this type of data analysis, data remains available through order of relativity to the query, thus providing productive information for predicting the impacts and make us alert to avoid certain vulnerable conditions. The main purpose of neural network data analytic is to gather, organize, categorize and provide the user with the best possible information that is available on World Wide Web. This paper has discussed about the research issues in neural network based covid-19 analytics possibility and also provided detailed review about the basic concepts of data analysis, neural networks and covid-19 scenario. Several open research issues and drawbacks which are exists in the current techniques are also discussed. This study and review would be helpful for researchers those who are doing their research in the domain of neural networks. The future scope of neural networks is to predict the user needs to improve the usability and scalability.

\section{REFERENCES}

1. Woo PC, Huang Y, Lau SK, Yuen KY. Corona virus genomics and bioinformatics analysis. Viruses 2010:18-20. 2. Drexler JF, GlozeRausch F, Glenda J, Cormen VM, MuthD, Goettsche M, et al. Genomic characterization of severe acute respiratory syndrome-related corona virus in European bats and classification of corona viruses based on partial RNAdependent RNA polymerase gene sequences. $J$ Virol 2010; 84:11336-49.

2. Yin $Y$, Wunderkindk RG. MERS, SARS and other corona viruses as causes of pneumonia. Respirology 2018; 23:130-7.

3. Peiris JSM, Lai ST, Poona L, et al. Corona virus as a possible cause of severe acute respiratory syndrome. The Lancet 2003; 361:1319-25.

4. Zaki AM, van Boheemen S, Bestebroer TM, Osterhaus AD, Fouchier RA. Isolation of a novel corona virus from a man with pneumonia in
Saudi Arabia. N Engl J Med 2012; 367:181420.

5. Seven days in medicine: 8-14 Jan 2020. BMJ 2020; 368:m132.31948945.

6. Imperial College London. Report 2: estimating the potential total number of novel corona virus cases in Wuhan City, China. Jan 2020. https://www.imperial.ac.uk/mrcglobalinfectiousdisease-analysis/news--wuhancoronavirus.

7. European Centre for Disease Prevention and Control data. Geographical distribution of 2019- nova cases. Available online :( https://www.ecdc.europa.eu/en/geographicaldistribution-2019-ncov-cases) (accessed on 05 February 2020)

8. World Health Organization, 2019- $n \mathrm{CoV}$ Situation Report-22 on 12 February, 2020. https://www.who.int/docs/defaultsource/coronav iruse/situation-reports/

9. Gralinski L, Menachery V. Return of the Corona virus: 2019-nCoV, Viruses 2020. 